\title{
ANÁLISIS TERMODINÁMICO DEL CICLO COMBINADO EN MODO HÍBRIDO DEL VALLE DE MÉXICO DE 384 MW A DIFERENTES CARGAS PARCIALES CONSIDERANDO CAÍDAS DE PRESIÓN Y DE TEMPERATURA
}

\author{
THERMODYNAMIC ANALYSIS OF THE COMBINED CYCLE IN THE \\ HYBRID MODE OF VALLE DE MEXICO OF 384 MW AT DIFFERENT \\ PARTIAL LOADS AND CONSIDERING PRESSURE AND TEMPERATURE \\ DROPS
}

\begin{abstract}
Eng. Sandra Marisol Sánchez Domínguez*, PhD. Helen Denise Lugo Méndez**, MSc. Israel Eliú Hernández Mora*, MSc. Edgar Vicente Torres González*, PhD. Raúl Lugo Leyte*
\end{abstract}

*Universidad Autónoma Metropolitana, Iztapalapa, Departamento de Ingeniería de Procesos e Hidráulica.

Avenida San Rafael Atlixco No.186, Colonia Vicentina, 09340, Iztapalapa, CDMX, México.

00-52-55-58044600-Ext.1156

E-mail: smsd@xanum.uam.mx, iehm@xanum.uam.mx, etorres@xanum.uam.mx, lulr@xanum.uam.mx.

**Universidad Autónoma Metropolitana, Cuajimalpa, Departamento de Procesos y Tecnología.

Av. Vasco de Quiroga No. 4871, Colonia Santa Fé, 05348, Cuajimalpa, CDMX, México. 00-52-55-58145500-Ext 3867

E-mail: hlugo@correo.cua.uam.mx.

Resumen: En este trabajo se evalúa el desempeño termodinámico de un ciclo combinado en modo híbrido a diferentes cargas, se realiza un análisis energético y exergético, considerando las caídas de presión y temperatura en las extracciones de vapor. Los resultados muestran que la cámara de combustión, el generador de vapor y la turbina de vapor son las fuentes principales de irreversibilidades y representan más del $38 \%$ de las pérdidas totales de exergía, donde la cámara de combustión presenta la mayor pérdida de exergía con $16.60 \%$. Las eficiencias térmica y exergética al $100 \%$ de carga son $58.79 \%$ y $77.40 \%$, respectivamente. Al reducir la carga a $75 \%$ y $50 \%$, la eficiencia térmica del ciclo disminuye $6.78 \%$ y $16.95 \%$ respectivamente. La relación entre el calor suministrado por el generador de vapor y el calor de los gases de escape de la turbina de gas es de 3.41 .

Palabras clave: Ciclo híbrido, Eficiencia, Exergía, Irreversibilidades

Abstract: In this work the thermodynamic performance of a combined cycle in hybrid
mode at different loads is evaluated, an energy and exergy analysis is performed,
considering the pressure and temperature drops in the steam extractions. The results show
that the combustion chamber, the steam generator and the steam turbine are the main
sources of irreversibilities and represent more than $38 \%$ of the total exergy losses, where
the combustion chamber represents the highest loss of exergy with $16.60 \%$. The thermal
and exergetic efficiencies of the cycle are $58.79 \%$ and $77.40 \%$, respectively. The 
combined cycle thermal efficiency decreases $6.78 \%$ and $16.95 \%$ when operating at a load of $75 \%$ and $50 \%$, respectively. The relation between the heat supplied by the steam generator and the Gas turbine combustion heat gases is 3.41.

Keywords: Hybrid cycle, Efficiency, Exergy, Irreversibilities

\section{INTRODUCCIÓN}

En los últimos años, la industria y las instituciones académicas han realizado un esfuerzo importante para el desarrollo eficiente y rentable de las centrales eléctricas. Se han investigado diferentes tecnologías, tales como energía solar, celdas de combustible, ciclos combinados, ciclos Rankine orgánicos, cogeneración, motores de combustión interna, arreglos híbridos, entre otros. Los ciclos combinados generan el $50 \%$ de energía eléctrica en México y es muy importante en los países donde el gas natural está disponible para la generación de electricidad. Como estos ciclos de potencia representan una manera atractiva de producir energía eléctrica a partir de gas natural, al hacer un análisis del desempeño, aplicando la primera y segunda ley de la termodinámica se obtienen los parámetros de desempeño, tales como eficiencia térmica, la destrucción de exergía y la eficiencia exergética de la planta (Kaushik, Reddy y Tyagi, 2011). Los tres componentes principales del ciclo combinado son: turbina de gas (TG); caldera de recuperación de calor (CRC); turbina de vapor (TV); sin embargo, la TG es el equipo que más influye en el desempeño, debido a la potencia que genera y a sus gases de combustión (Okoroigwe y Madhlopa, 2016). Las centrales de ciclo combinado, así como nucleares, solares, ciclos convencionales, entre otras, utilizan una sola fuente de calor. El usar más de una fuente de calor a diferentes temperaturas tiene ventajas en la eficiencia y en la reducción de emisiones y costos. Los sistemas híbridos de energía están relacionados con el concepto de repotenciación. Además, el sistema híbrido es aquel que utiliza múltiples fuentes de calor, ya sean de origen diferente o del mismo (Yue \& Lior, 2018). El mercado eléctrico siempre sigue las variaciones diarias, semanales y estacionales de la carga. Por lo tanto, existen fluctuaciones de carga en los sistemas de energía (Pinzon Ardila, Useche Gamboa, \& Rodriguez Galvis, 2011), que provocan: cambio de carga frecuente, operación a carga parcial; disminución de la eficiencia térmica de la central; así como emisiones de gases contaminantes, es decir, la calidad de la energía es la fuente de problemas de los sistemas de potencia (Garrido Arévalo, Díaz
Rodríguez, \& Pardo García, 2014). Las centrales que operan con gas natural presentan un rápido cambio de carga y un menor nivel de emisiones comparado con las carboeléctricas. Sin embargo, su eficiencia térmica de la central disminuye drásticamente con la reducción de la carga, además la variación de carga no es amplia (González, et al., 2018). Por lo tanto, predecir y estudiar las características de funcionamiento de las centrales de potencia que operan a carga parcial es de gran importancia. La regulación de la carga en las centrales termoeléctricas es por admisión parcial, mientras que en los ciclos combinados se realiza mediante presión deslizante, que consiste en regular la carga siguiendo a la presión de la caldera, ya que, de ese modo, las temperaturas de saturación se adaptan a las cargas en la caldera y la recuperación de calor es más eficiente.

\section{DESCRIPCIÓN DEL SISTEMA DEL CICLO COMBINADO EN MODO HÍBRIDO}

En este estudio se utilizan datos reales de funcionamiento del "paquete 4" en modo híbrido de la central del Valle de México, que es el acoplamiento de la unidad 4 (ciclo Rankine regenerativo con recalentamiento intermedio) y las unidades 5, 6 y 7 (ciclos de turbina de gas). Este modo de operación híbrido es el único ciclo aplicado en México, además de ser un ciclo de poca aplicación en el mundo, por la eficiencia que presentan. La central está ubicada en el municipio de Acolman, Estado de México, México y empezó a funcionar como ciclo híbrido en el año 2002. El combustible que se utiliza en las turbinas de gas es gas natural, sin embargo, en los generadores de vapor también se puede usar combustóleo. La Fig. 1 muestra el diagrama esquemático del ciclo combinado en modo híbrido, consta de tres TG; un ciclo de TV; tres CRC de dos presiones y un GV. Este ciclo está compuesto de 40 estados termodinámicos en el ciclo de vapor y 14 estados en el ciclo de gas (CFE, 2005). 


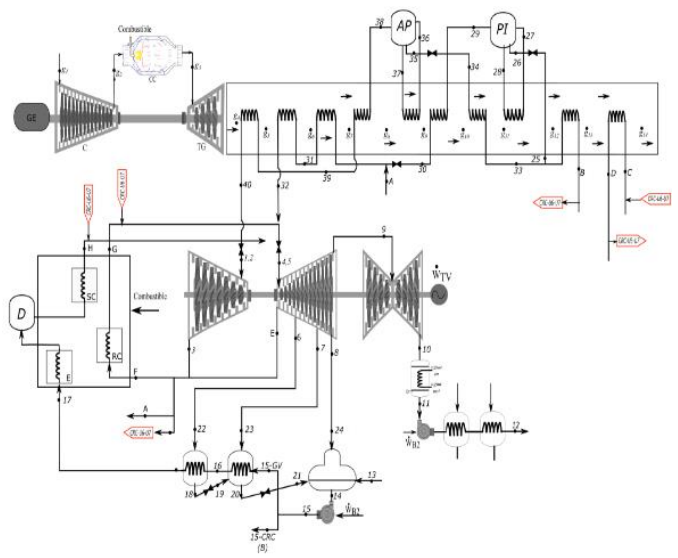

Fig. 1.Diagrama esquemático del ciclo híbrido

En la Tabla 1 se presentan las especificaciones técnicas de los principales equipos del ciclo

\section{Tabla 1: Especificaciones técnicas de los equipos principales del ciclo híbrido}

\begin{tabular}{lc}
\hline RELACIÓN DE PRESIONES DEL COMPRESOR & 15.5 \\
POTENCIA DE LA TURBINA DE GAS, MW & 84 \\
POTENCIA DE LA TURBINA DE VAPOR, MW & 300 \\
CAÍDA DE PRESIÓN EN LAS EXTRACCIONES, \% & 5 \\
CAÍDA DE TEMPERATURA EN LAS & 50 \\
EXTRACCIONES, \% & \\
TEMPERATURA DE VAPOR VIVO, ${ }^{\circ} \mathrm{C}$ & 526 \\
PRESIÓN DE VAPOR VIVO, bar & 166.5 \\
PRESIÓN DE CONDENSACIÓN, bar & 0.076 \\
TEMPERATURA DE CONDENSACIÓN, ${ }^{\circ} \mathrm{C}$ & 40.60 \\
\hline
\end{tabular}

\section{ANÁLISIS DE DESEMPEÑO}

En este trabajo se realiza un estudio termodinámico de un ciclo combinado en modo híbrido utilizando datos reales de la central (CFE, 2005). También se realiza un análisis paramétrico a diferentes cargas de operación. Para el estudio de desempeño termodinámico de la central, se realiza un análisis energético y un análisis exergético. Para los cálculos de los estados termodinámicos, se hacen las siguientes suposiciones:

- Los productos de combustión y el aire se comportan como gas ideal.

- La composición molar del aire es $78 \%$ de $\mathrm{N}_{2}$ y $21 \%$ de $\mathrm{O}_{2}$.

- La composición del combustible es $88 \%$ de $\mathrm{CH}_{4}, 9 \%$ de $\mathrm{C}_{2} \mathrm{H}_{6}$ y $3 \%$ de $\mathrm{C}_{3} \mathrm{H}_{8}$, con un PCI de $49,383.57 \mathrm{~kJ} / \mathrm{kg}$.

- Las condiciones del estado muerto son $P_{0}=1$ bar y $T_{0}=273.15 \mathrm{~K}$.

- No existen pérdidas de calor en los equipos del ciclo.

Como resultado de las consideraciones antes mencionadas, la composición de los gases de combustión obtenida de la cámara de combustión es $6.87 \%$ de $\mathrm{CO}_{2} ; 5.26 \%$ de $\mathrm{H}_{2} \mathrm{O} ; 74.88 \%$ de $\mathrm{N}_{2}$; y $12.99 \%$ de $\mathrm{O}_{2}$. La relación aire-combustible, rac, es $39.61 \mathrm{~kg}_{\mathrm{a}} / \mathrm{kg}_{\text {comb }}$ y un exceso de aire de $135.2 \%$. A continuación, se presentan los métodos de análisis de desempeño, se realiza un análisis a diferentes cargas y los resultados se comparan con los resultados operativos reales.

\subsection{Ciclo de gas}

El estudio energético del ciclo combinado en modo híbrido se lleva a cabo al dividir el sistema en los ciclos de gas y de vapor. Se realizan balances de masa y energía a cada equipo de la central, para obtener los estados del ciclo. El cambio de entropía de cada uno de los estados del ciclo, se obtiene con la siguiente expresión:

$s_{i}=s_{0}+c_{P_{i}} \ln \left(T_{i} / T_{0}\right)-R_{i} \ln \left(P_{i} / P_{0}\right)$

Los ciclos de gas operan con el ciclo termodinámico Joule-Brayton para producir energía mecánica. Los equipos principales de este ciclo son: compresor (C), cámara de combustión (CC) y turbina de gas. El ciclo de gas abierto es más utilizado, debido a la capacidad que tiene para alcanzar temperaturas altas a la entrada de la turbina, haciendo más eficiente al ciclo, más compacto y menos costoso (Olumayegun, et al., 2016). En la central de ciclo combinado en modo híbrido, el aire entra al compresor a temperatura ambiente, después de la compresión, el aire comprimido entra a la cámara de combustión, donde, junto con el gas natural, se lleva a cabo la combustión; en consecuencia, la temperatura de los gases de combustión alcanzan $1,300{ }^{\circ} \mathrm{C}$ (CFE, 2005). Los gases de combustión se expanden en la $\mathrm{TG}$, y salen de ésta entre $538{ }^{\circ} \mathrm{C}$ y $600{ }^{\circ} \mathrm{C}$. La Fig. 2 muestra el diagrama temperatura-entropía $(T-s)$ del ciclo de la turbina de gas y de los gases de combustión $\left(g_{4}-g_{14}\right)$, estos gases de combustión entran a la CRC a $674{ }^{\circ} \mathrm{C}$, después de ceder su energía al agua de alimentación, estos gases salen al medio ambiente a $163.41^{\circ} \mathrm{C}$. A continuación, se describen las ecuaciones para obtener los parámetros de desempeño. 


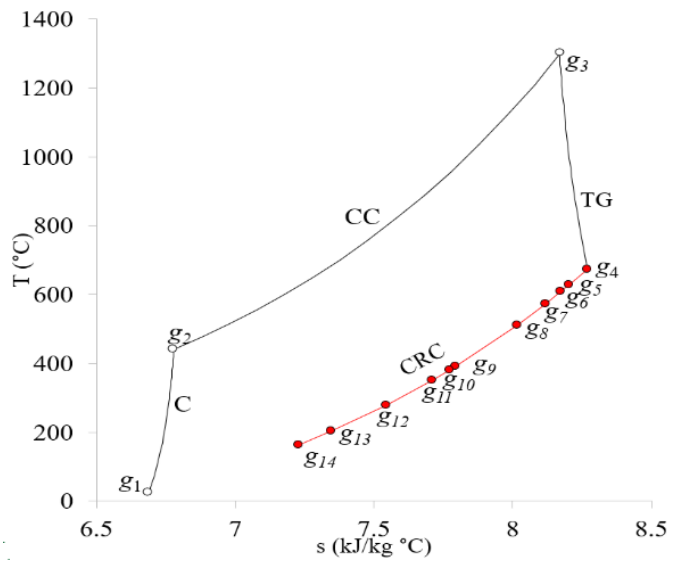

Fig. 2. Diagrama $T$-s de la $T G$

La temperatura del aire después de la compresión es

$$
T_{g_{2}}=T_{g_{1}}\left[1+\left(1 / \eta_{\text {SIC }}\right)\left(\pi_{C}^{x_{a}}-1\right)\right]
$$

La potencia suministrada al compresor es

$$
\dot{W}_{C}=\frac{\dot{m}_{a} c_{P_{a}} T_{g_{1}}}{\eta_{\text {SIC }}}\left(\pi_{C}^{x_{a}}-1\right)
$$

El flujo de calor suministrado a la turbina de gas

$$
\dot{Q}_{\text {sumTG }}=\dot{m}_{a} c_{P_{a}} T_{g_{1}}\left[(r c a+1) \frac{c_{P_{s c}}}{c_{P_{a}}} y-1-\frac{1}{\eta_{S I C}}\left(\pi_{C}^{x_{a}}-1\right)\right]
$$

La temperatura de los gases de combustión se expresa como

$T_{g_{4}}=T_{g_{3}}\left\{1-\eta_{S I T}\left[1-\left(1 / \pi_{C}{ }^{x_{g c}}\right)\right]\right\}$

La potencia generada por TG se expresa como

$$
\dot{W}_{T G}=\dot{m}_{a} c_{P_{g c}}(1+r c a)\left\{T_{g_{3}} \eta_{S I T}\left[1-\left(1 / \pi_{C}^{x_{g c}}\right)\right]\right\}
$$

El trabajo motor del ciclo de gas se obtiene como

$w_{M}=c_{P_{a}} T_{g_{1}}\left\{\left[\frac{c_{P_{s c}}}{c_{P_{a}}}(1+r c a) \frac{T_{g_{3}}}{T_{g_{1}}} \eta_{s i t}\left(1-\frac{1}{\pi_{C}^{x_{c i}}}\right)\right]-\left[\frac{1}{\eta_{s i c}}\left(\pi_{C}^{x_{\text {sic }}}-1\right)\right]\right\}$

Los flujos másicos de aire y combustible utilizados en el ciclo de gas, se calculan respectivamente con

$\dot{m}_{a}=\dot{W}_{M} / w_{M}$

$\dot{m}_{\text {comb }}=\dot{m}_{a} / \mathrm{rac}$

El flujo másico de gases de combustión es la suma del flujo de aire y combustible que entran a la CC.

$\dot{m}_{g c}=\dot{m}_{\text {comb }}+\dot{m}_{a}$

La eficiencia térmica del ciclo de gas se expresa como

$\eta_{T G}=\left(\dot{W}_{T G}-\dot{W}_{C}\right) / \dot{Q}_{\text {sumTG }}$

Para realizar un análisis exergético e identificar la fuente, lugar y magnitud de las irreversibilidades, primero se debe entender que la exergía se define como el máximo trabajo que se puede producir en un proceso o un sistema cuando se alcanza un equilibrio con el entorno de referencia. Este tipo de análisis se aplica en varios procesos y sistemas, para evaluarlos y mejorarlos (Gupta, y otros, 2015). La exergía de flujo para cada estado del ciclo de gas y vapor, se expresa como la suma de la exergía química y física (Lara, et al., 2017), en este trabajo se desprecia la exergía cinética, exergía potencial y la exergía química, es decir, se calcula como

$$
\dot{E}^{f i s}=\dot{m}\left[\left(h-h_{0}\right)-T_{0}\left(s-s_{0}\right)\right]
$$

Un balance exergético de un dispositivo del ciclo combinado se expresa de la siguiente manera.

$$
\dot{E}_{Q}+\sum \dot{E}_{e}=\sum \dot{E}_{s}+\dot{W}+\dot{I}
$$

Para el proceso de compresión, la irreversibilidad es

$$
\dot{I}_{C}=\dot{E}_{g_{1}}+\dot{W}_{C}-\dot{E}_{g_{2}}
$$

La exergía química del combustible se expresa de la siguiente manera

$$
\widetilde{\varepsilon}_{0_{\text {mezcla }}}=\left[\sum_{i} x_{i} \widetilde{\varepsilon}_{0 i}+R T_{0} \sum_{i} x_{i} \ln \left(x_{i}\right)\right]
$$

donde, $x_{i}$, es la fracción molar de los componentes. Otra forma de obtener la exergía del combustible es

$$
\dot{E}_{\text {comb }}=\dot{m}_{\text {comb }} P C I_{\text {comb }} \xi=\dot{W}_{\text {máx }}
$$

donde el término $\xi$ es el cociente de la exergía química del combustible y el poder calorífico del mismo, este término se obtiene con la ecuación (17) a partir del número de carbonos, $n$, e hidrógenos, $m$, (Kotas, 1985).

$\xi=1.033+0.0169(m / n)-(0.0698 / n)$

La exergía química para un gas ideal se expresa de la siguiente manera

$\widetilde{\varepsilon}_{i}=R T_{0} \ln \left(P_{i} / P_{0}\right)$

donde $P_{i}$ es la presión parcial del compuesto y $P_{0}$, la presión de referencia.

La irreversibilidad de la cámara de combustión se expresa como

$\dot{I}_{C C}=\dot{W}_{\max }+\left(\dot{E}_{g_{2}}-\dot{E}_{g_{3}}\right)-\dot{E}_{Q}$

donde $\dot{E}_{Q}$ es la exergía debido a la transferencia de calor.

La irreversibilidad para el proceso de expansión se expresa de la siguiente manera

$$
\dot{I}_{T G}=\dot{E}_{g_{3}}-\dot{E}_{g_{4}}-\dot{W}_{T G}
$$

La eficiencia exergética del ciclo de gas se escribe como (Fallah, et al., 2016): 
$\psi_{T G}=\dot{W}_{T G} / \dot{E}_{c o m b}$

La Fig. 3 muestra los estados del ciclo de gas y de los gases de combustión. El proceso de combustión presenta el mayor incremento de exergía con 1,021 $\mathrm{kJ} / \mathrm{kg}$. Así también, muestra que los gases de combustión salen de la turbina con una exergía de $819 \mathrm{~kJ} / \mathrm{kg}$ y con $402.72 \mathrm{~kJ} / \mathrm{kg}$ de la CRC.

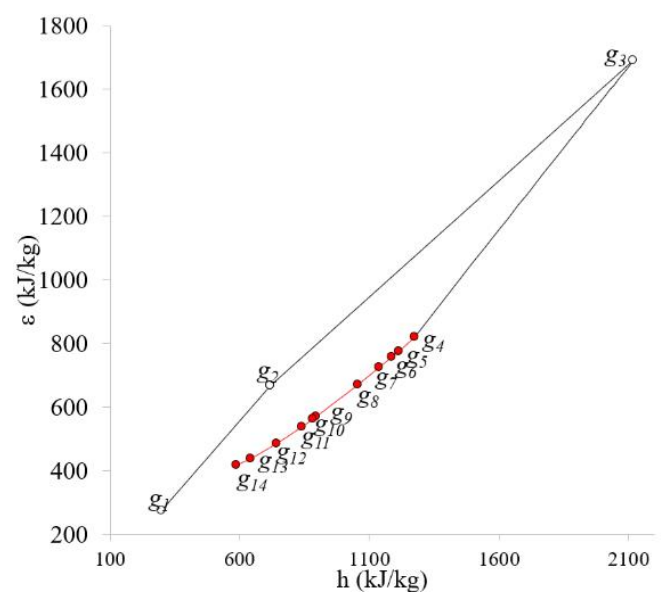

Fig. 3. Diagrama $\varepsilon$-h de la $T G$

\subsection{Ciclo de vapor}

Este ciclo opera con el ciclo termodinámico Rankine regenerativo con recalentamiento. En esta sección, se describen las ecuaciones para el análisis energético del ciclo. La TV es un dispositivo útil para convertir la energía térmica en energía mecánica. Además, otro beneficio de los sistemas de turbinas de vapor es que poseen la capacidad de usar distintos tipos de combustibles en el GV y también se pueden usar distintos fluidos de trabajo como fluidos orgánicos. La eficiencia térmica de una planta moderna de energía de ciclo Rankine puede alcanzar más del $40 \%$ y se puede aumentar mediante el uso de opciones combinadas o integradas de ciclo y/o subsistemas de recuperación de calor (Salisbury, 1950; Mohammad y McGowan, 2018). El calor suministrado por el GV se obtiene del prontuario de la central, éste cambia según la carga a la que se opere, por ejemplo: al $100 \%$ de carga se suministra $454 \mathrm{MW}$, y al operar al $75 \%$ y $50 \%$ se suministra 417 y 410 MW, respectivamente. Por otra parte, el calor rechazado en el condensador se expresa como

$\dot{Q}_{\text {cond }}=\dot{m}_{v_{10}}\left(h_{v_{10}}-h_{v_{11}}\right)$

En la CRC, las diferencias de temperaturas de pinch point para los evaporadores son de gran importancia, a partir de éstos se obtiene la temperatura del estado $g_{9}$ y $g_{12}$ del ciclo de gas, éstos se expresan como

$$
\begin{aligned}
& \Delta T_{P P, P I}=T_{g_{12}}-T_{v_{27}} \\
& \Delta T_{P P, A P}=T_{g_{9}}-T_{v_{36}}
\end{aligned}
$$

El flujo másico de vapor que se produce para trabajar las turbinas es

$$
\dot{m}_{\text {vapor }}=\frac{\dot{W}_{T V}}{w_{M}}=\frac{\dot{W}_{T V}}{\left|w_{T V}\right|-\left|w_{B}\right|}
$$

El flujo másico de combustible quemado en el GV se obtiene como

$$
\dot{m}_{\text {comb }, G V}=\left(\dot{Q}_{\text {sum }, G V}\right) / P C I
$$

Los balances de masa y energía de los equipos que conforman la CRC son de gran importancia, ya que con éstos se obtienen los flujos de vapor que se extraen en la TVPI y se obtiene la energía cedida al vapor de agua. Estos balances por equipo se expresan como:

$$
\begin{aligned}
& \text { SCAP: } c_{P_{g c}} \dot{m}_{g c}\left(T_{g_{4}}-T_{g_{5}}\right)=\dot{m}_{V_{39}}\left(h_{V_{40}}-h_{V_{39}}\right) \\
& \text { SCPI3: } c_{P_{g c}} \dot{m}_{g c}\left(T_{g_{5}}-T_{g_{6}}\right)=\dot{m}_{V_{32}}\left(h_{V_{32}}-h_{V_{31}}\right) \\
& \text { SCPI2: } c_{P_{g c}} \dot{m}_{g c}\left(T_{g_{6}}-T_{g_{7}}\right)=\left(\dot{m}_{V_{31}}+\dot{m}_{A}\right)\left(h_{V_{31}}-h_{V_{30}}\right) \\
& \text { SCAP1: } c_{P_{g c}} \dot{m}_{g c}\left(T_{g_{7}}-T_{g_{8}}\right)=\dot{m}_{V_{38}}\left(h_{V_{39}}-h_{V_{38}}\right) \\
& \text { EVAP: } c_{P_{g c}} \dot{m}_{g c}\left(T_{g_{8}}-T_{g_{9}}\right)=\dot{m}_{V_{36}}\left(h_{V_{37}}-h_{V_{36}}\right) \\
& \text { SCPI1: } c_{P_{g c}} \dot{m}_{g c}\left(T_{g_{9}}-T_{g_{10}}\right)=\dot{m}_{V_{29}}\left(h_{V_{30}}-h_{V_{29}}\right) \\
& \text { ECAP2: } c_{P_{g c}} \dot{m}_{g c}\left(T_{g_{10}}-T_{g_{11}}\right)=\dot{m}_{V_{34}}\left(h_{V_{34}}-h_{V_{33}}\right) \\
& \text { EVPI: } c_{P_{g c}} \dot{m}_{g c}\left(T_{g_{11}}-T_{g_{12}}\right)=\dot{m}_{V_{27}}\left(h_{V_{28}}-h_{V_{27}}\right) \\
& \text { ECAP1: } c_{P_{g c}} \dot{m}_{g c}\left(T_{g_{12}}-T_{g_{13}}\right)=\dot{m}_{V_{15 C R c}}\left(h_{V_{25}}-h_{V_{15 C R c}}\right) \\
& \text { ECBP: } c_{P_{g c}} \dot{m}_{g c}\left(T_{g_{13}}-T_{g_{14}}\right)=\dot{m}_{V_{12}}\left(h_{V_{13}}-h_{V_{12}}\right)
\end{aligned}
$$

La potencia generada por la TV se expresa como

$$
\dot{W}_{T V}=\dot{W}_{T V A P}+\dot{W}_{T V P I}+\dot{W}_{T V B P}
$$

El trabajo suministrado a las bombas se expresa como

$\dot{W}_{B}=\dot{m}_{V 11}\left(h_{V_{12}}-h_{V_{11}}\right)+\dot{m}_{V 14}\left(h_{V_{15}}-h_{V_{14}}\right)$

La potencia motor es la resta de la potencia de la turbina y de la bomba

$\dot{W}_{M}=\left|\dot{W}_{T V}\right|-\left|\dot{W}_{B}\right|$

El flujo de calor suministrado al ciclo de vapor es

$\dot{Q}_{\text {Sum }_{T V}}=\dot{Q}_{\text {sum }_{G V}}+\dot{Q}_{\text {sum }_{C R C-U 5}}$ 
Eficiencia térmica del ciclo de la turbina de vapor

$\eta_{T V}=\dot{W}_{T V} / \dot{Q}_{\text {sumTV }}$

La Fig. 4 muestra como el ciclo de vapor aprovecha la energía de los gases de combustión provenientes de la TG, mientras se hacen pasar por la CRC, donde calientan, evaporan y sobrecalientan el $43 \%$ del flujo de agua de alimentación y el $57 \%$ restante, se envía al GV para ser precalentado. Todo el vapor producido por estos equipos, se utiliza en la TV para producir 300 MW, para cerrar el ciclo, un condensador convierte el vapor húmedo de la salida de la turbina en líquido saturado, para bombearlo y llevarlo hasta el generador de vapor. En el proceso de condensación se pierde el $89 \%$ de la energía suministrada al ciclo de vapor.

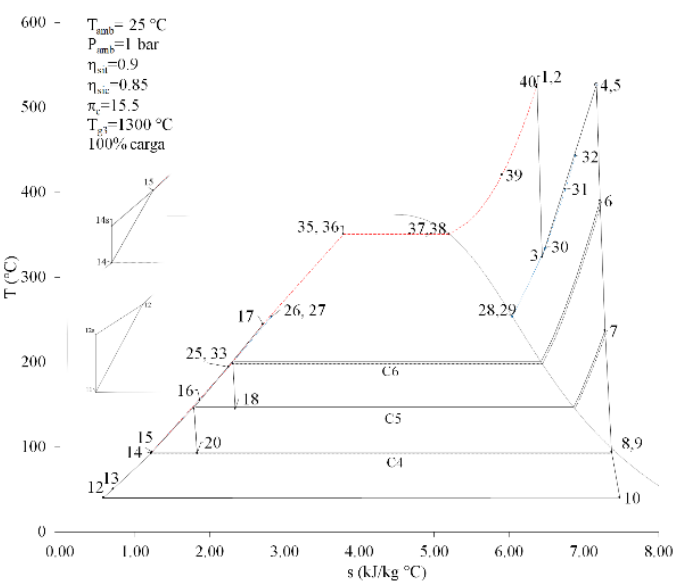

Fig. 4. Diagrama T-s del ciclo de vapor

Las irreversibilidades de los equipos del ciclo de vapor se expresan como

$$
\begin{aligned}
& \mathrm{CRC}: \dot{\mathrm{E}}_{v_{15 C R C}}+\dot{E}_{g_{4}}+\dot{E}_{A}-\dot{\mathrm{E}}_{v_{40}}-\dot{\mathrm{E}}_{v_{32}}-\dot{E}_{g_{14}} \\
& \mathrm{GV}: \dot{\mathrm{E}}_{v_{17}}+\dot{\mathrm{E}}_{F}+\dot{\mathrm{E}}_{c o m b}-\dot{\mathrm{E}}_{H}-\dot{\mathrm{E}}_{G} \\
& \mathrm{TV}: \dot{E}_{v_{2}}-\dot{E}_{v_{3}}-\dot{W}_{T V, A P}+\dot{\mathrm{E}}_{v_{5}}+\dot{E}_{E}-\dot{\mathrm{E}}_{v_{6}}-\dot{\mathrm{E}}_{v_{7}} \\
& -\dot{\mathrm{E}}_{v_{8}}-\dot{\mathrm{E}}_{v_{9}}-\dot{W}_{T V, P I}+\dot{\mathrm{E}}_{v_{9}}-\dot{\mathrm{E}}_{v_{10}}-\dot{W}_{T V, B P} \\
& \text { Cond: } \dot{\mathrm{E}}_{v_{10}}+\dot{\mathrm{E}}_{a g u a_{i n}}-\dot{\mathrm{E}}_{v_{11}}-\dot{\mathrm{E}}_{\text {agua }} \\
& \text { Bomba: } \dot{\mathrm{E}}_{v_{11}}+\dot{W}_{B 1}-\dot{\mathrm{E}}_{v_{12}}+\dot{\mathrm{E}}_{v_{14}}+\dot{W}_{B 2}-\dot{\mathrm{E}}_{v_{15}}
\end{aligned}
$$

La eficiencia exergética del ciclo de vapor se expresa como

$\psi_{T V}=\dot{W}_{T V} / \dot{E}_{c o m b, G V}$

El flujo de calor suministrado, la potencia generada del ciclo híbrido se expresan respectivamente como

$$
\begin{aligned}
& \dot{Q}_{\mathrm{CH}}=\dot{Q}_{\text {sumTV }}+3 \dot{Q}_{\text {sumTG }} \\
& \dot{W}_{C H}=\dot{W}_{M}+3 \dot{W}_{T G}
\end{aligned}
$$

La eficiencia térmica de ciclo combinado en modo híbrido se expresa como

$\eta_{C H}=\left(\eta_{T G}+\eta_{T V}-\eta_{T G} \eta_{T V}+\beta \eta_{T V}\right) /(1+\beta)$

donde $\beta$ es la relación del calor suministrado por el GV y por la CRC.

$\beta=\dot{Q}_{\text {sum }, G V} / \dot{Q}_{\text {sum }, C R C}$

La eficiencia exergética del ciclo combinado en modo híbrido, se expresa de la siguiente manera

$\psi_{C H}=\dot{W}_{C H} / \dot{E}_{\text {comb }}$

La Fig. 5 muestra que la menor pérdida de exergía se tiene en el proceso de condensación, y aunque la energía que se pierde en este equipo es alta debido a que la calidad es baja y no se puede aprovechar, ya que está cerca del estado de referencia. El vapor a la entrada de la TVAP presenta la mayor exergía del ciclo.

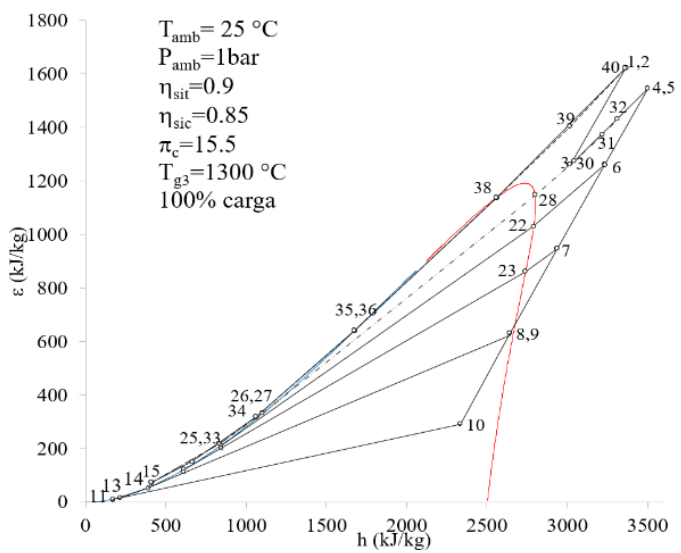

Fig. 5. Diagrama $\varepsilon$-h del ciclo de vapor

\section{RESULTADOS}

La Fig. 6 muestra el perfil de temperaturas de la CRC, donde se representa el intercambio de energía en forma de calor de los gases de combustión y el agua de alimentación. Se muestra que los gases entran a una temperatura de $674{ }^{\circ} \mathrm{C}$ y disminuyen mientras pasa por cada equipo hasta llegar a $163.41{ }^{\circ} \mathrm{C}$, aproximadamente. El agua de alimentación entra a contracorriente de los gases a una temperatura de $40{ }^{\circ} \mathrm{C}$. En el economizador y evaporador se tiene la mayor transferencia de calor. El cambio de temperatura de pinch point de alta e intermedia presión, es $41.73{ }^{\circ} \mathrm{C}$ y $25.58{ }^{\circ} \mathrm{C}$, respectivamente. La temperatura de aproximación caliente tiene un valor de $150{ }^{\circ} \mathrm{C}$. 


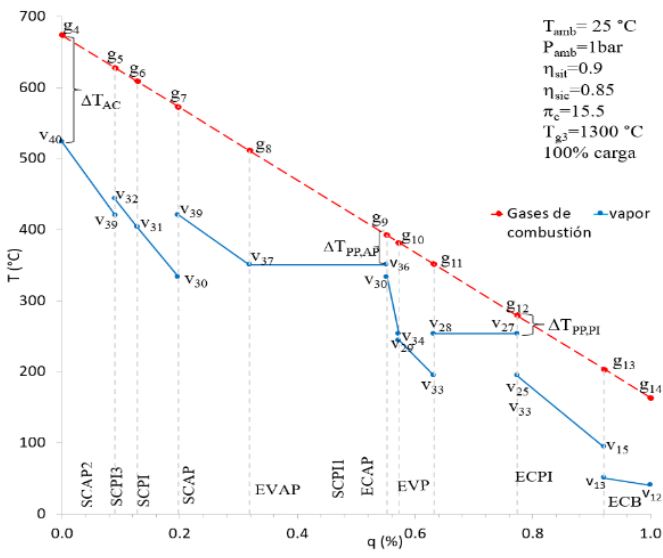

Fig. 6. Perfil de temperaturas de la CRC

La Fig. 7 muestra que la eficiencia térmica del ciclo de gas es de $30.66 \%$ al $100 \%$ de carga, mientras que en el ciclo de vapor es $51.10 \%$; sin embargo, al trabajar como ciclo híbrido la eficiencia térmica de la central aumenta hasta $54.92 \%$. La Fig. 8 muestra que la eficiencia térmica del ciclo híbrido se ve afectada por el factor $\beta$. Al trabajar al $100 \%$ de carga, se tiene un valor de $\beta=3.4131$, es decir, la fuente que aporta más calor es el GV. Este factor decrece conforme la carga se reduce, así como la eficiencia térmica y exergética del ciclo. Al operar al $100 \%$ de carga, se tiene una eficiencia térmica de $54.50 \%$ y considerando pérdidas en la CRC es $59.42 \%$, mientras que la eficiencia exergética es de 77.40 $\%$. Con relación al $100 \%$ de carga, la eficiencia térmica disminuye $6.78 \%$ y $16.95 \%$ al operar al $75 \%$ y $50 \%$ de carga, respectivamente, mientras que la exergética disminuye $2.55 \%$ y $10.16 \%$, respectivamente.

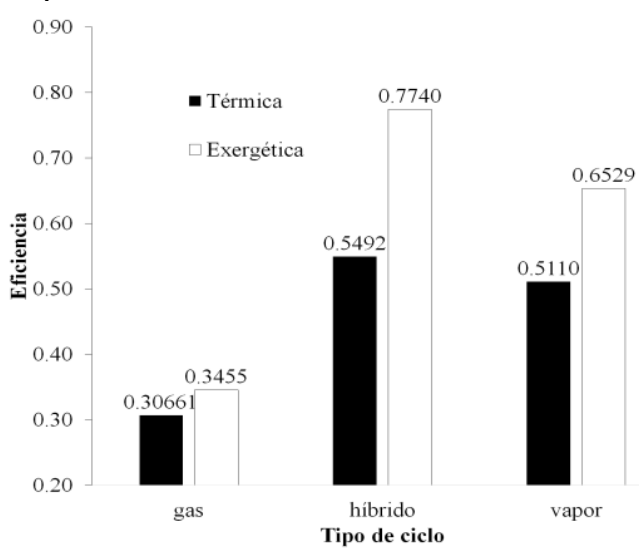

Fig. 7. Eficiencia térmica y exergética de los ciclos que conforman al ciclo combinado híbrido

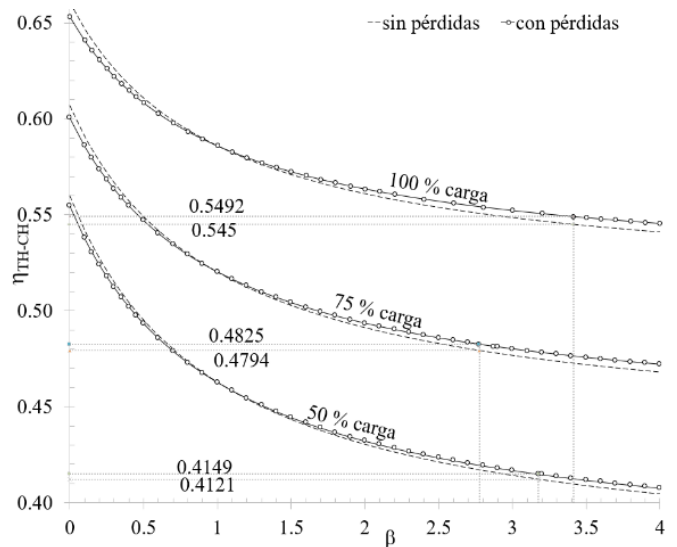

Fig. 8. Eficiencia térmica de ciclo combinado en modo híbrido para diferentes $\beta$

La Fig. 9 muestra el porcentaje de irreversibilidades generadas en los equipos con relación al flujo de exergía del combustible. Los equipos donde se tiene una reacción de combustión, son los que aportan mayores irreversibilidades, $28.82 \%$. El $16.61 \%$ corresponden a la CC, el $14 \%$ son pérdidas inevitables debido a la oxidación del combustible. Los equipos con menos irreversibilidades, $0.67 \%$, son los calentadores $(\mathrm{Cal})$, la bomba y el compresor. Las irreversibilidades de los equipos representan el $41.66 \%$, es decir, más de 600 MW. Esta exergía perdida disminuye $6.15 \%$ y $10.85 \%$ al operar al $75 \%$ y $50 \%$ de carga, con respecto al $100 \%$ de carga.

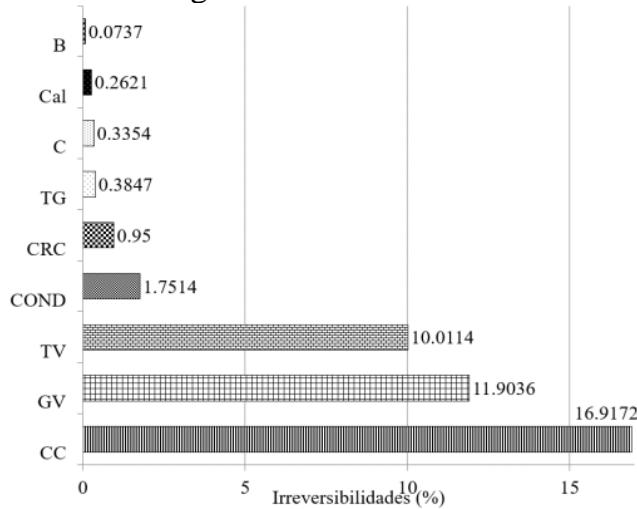

Fig. 9. Porcentaje de irreversibilidades en los equipos del ciclo combinado en modo híbrido

\section{CONCLUSIONES}

Los resultados muestran que contar con las dos fuentes de calor, presenta ventajas respecto al ciclo de gas y el ciclo de vapor, debido a que se tiene un aumento en la eficiencia térmica.

La cámara de combustión, el generador de vapor y la turbina de vapor son los equipos que más 
contribuyen a las irreversibilidades, por lo tanto, también tienen un gran impacto en la eficiencia térmica total de la central.

Operar el ciclo a $50 \%$ y $75 \%$ de carga provoca la disminución de la eficiencia térmica y exergética, además de cantidades altas de energía.

En esta unidad, no se hace una cogeneración de los gases de combustión del GV, sin embargo, si se llega a utilizar ese flujo de gases de combustión para precalentar el agua de alimentación, se tendrá un aumento en la eficiencia térmica de la central, y se reducirá el impacto ambiental.

Finalmente, este trabajo se puede complementar con un análisis exergoeconómico-ambiental, con el objetivo de mejorar el análisis de desempeño de esta central y así, mejorar en el conocimiento de alternativas para la disminución del impacto ambiental.

\section{RECONOCIMIENTO}

Se agradece a la Maestría en Ciencias (Energía y Medio Ambiente) de la Universidad Autónoma Metropolitana, Unidad Iztapalapa, la cual, pertenece al Padrón Nacional de Posgrados de Calidad del CONACyT, por la beca recibida. De la misma manera se agradece a los laboratorios PP2 y T041 por todo el apoyo.

\section{REFERENCIAS}

CFE. (2005). Prontuario de la Central Termoeléctrica de Ciclo Híbrido "Valle de México". Comisión Federal de Electricidad, México.

C. J. Torres, J. F. Archila, M. L. Tronco, M. Becker, A. J. V. Porto, A. J. Tiberti. (2013). Estudio cinemático de una plataforma robótica para agricultura. REVISTA COLOMBIANA DE TECNOLOGÍAS DE AVANZADA, ISSN: 1692-7257. 2(22).

Fallah, M., Siyahi, H., Akbarpour Ghiasi, R., Mahmoudi, S., Yaria, M., \& Rosen, M. (2016). "Comparison of different gas turbine cycles and advanced exergy analysis of the most effective". Energy, Vol. 116, No. 1.

Garrido Arévalo, V. M., Díaz Rodríguez, J. L., \& Pardo García, A. (2014). "Clasificadores inteligentes para determinar la afectacion a la calidad de la enegía eléctrica”. Revista colombiana de tecnologías de avanzada, Vol.1. No.23.

González Salazar, M. A., Kirsten, T., \& Prchlik, L. (2018). "Review of the operational flexibility and emissions of gas- and coal-fired power plants in a future with growing renewables".
Renewable and Sustainable Energy Reviews, Vol. 82.

Gupta, M., Kaushik, S., Ranjan, K., Reddyd, S., Tyagie, S., \& Panwarc, N. (2015). "Thermodynamic performance evaluation of solar and other thermal power generation systems: A review". Renewable and Sustainable Energy Reviews, Vol 50.

Kaushik, S., Siva Reddy, V., \& Tyagi, S. (2011). "Energy and exergy analyses of thermal power plants: A review". Renewable and Sustainable Energy Reviews, Vol 15.

Kotas, T. (1985). The exergy method of thermal plant analysis. Florida: Butterworth-Heinemann .

Lara, Y., Petrakopoulou, F., Morosuk, T., Boyano, A., y Tsatsaronis, G. (2017). “An exergy-based study on the relationship between costs and environmental impacts in power plants". Energy, Vol. 138.

Mohammad, K., y McGowan, J. G. (2018). "Thermodynamic analysis of hybrid cycles based on a regenerative steam Rankine cycle for cogeneration and trigeneration". Energy Conversion and Management, Vol. 158.

Okoroigwe, E., y Madhlopa, A. (2016). "An integrated combined cycle system driven by a solar tower: A review". Renewable and Sustainable Energy Reviews, Vol. 57.

Olumayegun, O., Wang, M., \& Kelsall, G. (2016). "Closed-cycle gas turbine for power generation: A state-of-the-art review". Fuel, Vol. 180.

Pinzón Ardila, O., Useche Gamboa, M. I., \& Rodriguez Galvis, W. L. (2011). "Diseño e implementacion de un equipo analizador de calidad de energía eléctrica". Revista colombiana de Tecnologías de Avanzada,Vol.2, No.26.

Salisbury, J. K. (1950). Steam Turbines and Their Cycles. New York: Wiley.

Torres, C., Archila, J., Tronco, M., Becker, M., Viera Porto, A., \& Tiberti, A. (2013). Estudio cinemático de una plataforma robótica para agricultura. REVISTA COLOMBIANA DE TECNOLOGÍAS DE AVANZADA, 2(22)

Yue, T., \& Lior, N. (2018). "Thermodynamic analysis of hybrid Rankine cycles using multiple heat sources of different temperatures". Applied energy, Vol. 222. 